International Conference Mathematical and Computational Biology 2011 International Journal of Modern Physics: Conference Series

Vol. 9 (2012) 59-67

(C) World Scientific Publishing Company

DOI: $10.1142 / \mathrm{S} 2010194512005107$

\title{
A SIX-LINK KINEMATIC CHAIN MODEL OF HUMAN BODY USING KANE'S METHOD
}

\section{A. S. RAMBELY}

School of Mathematical Sciences, Faculty of Science and Technology, Universiti Kebangsaan Malaysia, Bangi, 43600 Selangor, Malaysia asr@ukm.my

FAZROLROZI

Biomechanics Research Group, Biomechanics and Simulation Laboratory, School of Mathematical Sciences, Faculty of Science and Technology, Universiti Kebangsaan Malaysia, 43600 Selangor, Malaysia fazrolrozi@yahoo.com

\begin{abstract}
A biomechanics model of six-link kinematic chain of human body is developed by using Kane's method. The kinematic data comprise of six segments; foot, calf, thigh, trunk, upper arm and forearm, are obtained through data collection of walking, running and jumping using the Vicon Nexus system. The motion capture system uses 12 Vicon MX-3+ cameras and 12 Vicon MX-F40 cameras, two DV $(50 \mathrm{~Hz})$ cameras and a force plate $(100 \mathrm{~Hz})$. Inverse dynamics approach is used to obtain the unknown value of torques produced by joint segments during walking, running and jumping activities. The results show that the largest value of torques produced occurs at the foot segment.
\end{abstract}

Keywords: Kane's method; biomechanics model; kinematic chain; inverse dynamics.

\section{Introduction}

Kane's method has been applied in research into robotic movement ${ }^{1-2}$, modelling surgical repairs of hip joints ${ }^{3}$, and sports fields ${ }^{4-5}$. We construct a two-dimensional six-link kinematic chain model of the human body. The six-links are the foot, calf, thigh, trunk, upper arm and forearm, Fig. 1. An inverse dynamic method (Kane's method) is used to estimate the torques causing rotational movements in each joint. The model is applied to human movement. 


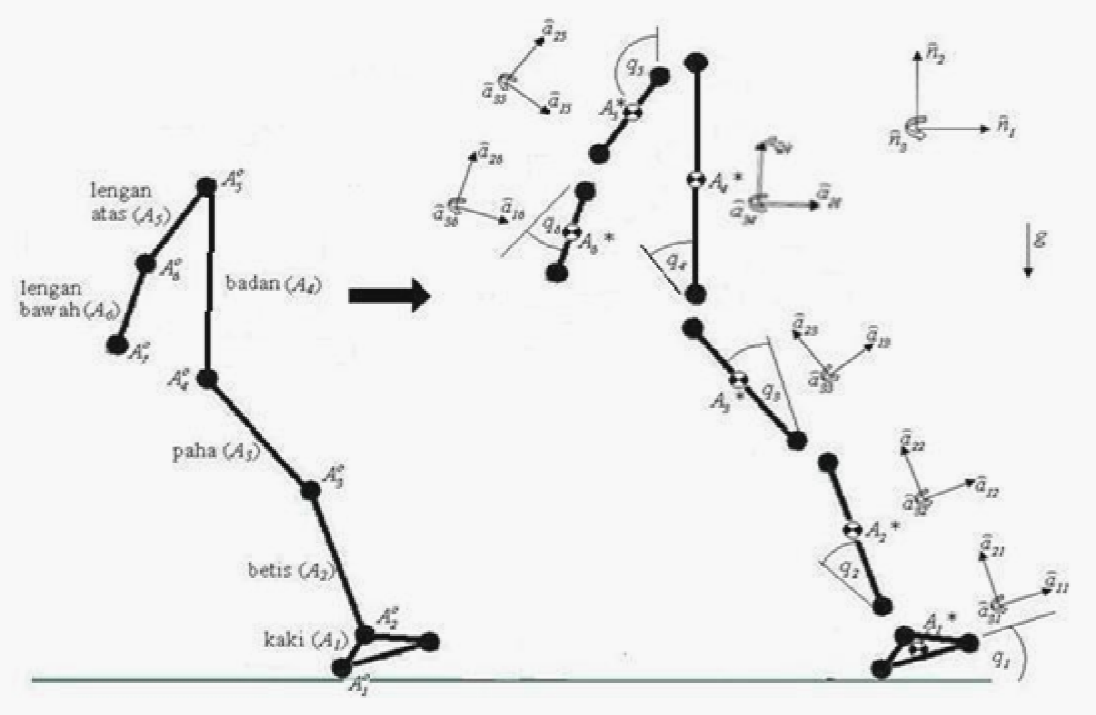

Fig. 1 Kinematics chain of human body

\section{Methodology}

\subsection{Mathematical modeling}

Modeling via Kane's approach starts with determining the angular velocity, angular acceleration, linear velocity and linear acceleration for each segment, $A_{i},(i=1,2, \ldots, 6)$ with respect to reference frame $N$. Angular velocities are determined to be,

$$
{ }^{N} \vec{\omega}^{A_{i}}=\left(\sum \dot{q}_{i}\right) \hat{a}_{3 i}
$$

where $q$ is an angular rotation of body $A_{i}$, thus $\dot{q}$ is its angular speed and $\hat{a}_{A i}$ is the mutually perpendicular unit vectors which define vector component directions of each rigid body reference frames, $A_{i}$. Similarly the angular accelerations are,

$$
{ }^{N} \vec{\alpha}^{A_{i}}=\left(\sum \ddot{q}_{i}\right) \hat{a}_{3 i} .
$$

Linear velocity of segment, $A_{i}$ with respect to reference frame $N$ is,

$$
{ }^{N} \vec{v}^{A_{i}^{o}}=\sum l_{A_{i-1}} \dot{q}_{i} \widehat{a}_{l i}
$$

where $l_{A}$ is the length of each segment and

$$
{ }^{N} \vec{v}^{A_{i}^{*}}=\sum l_{A_{i-1}} \dot{q}_{i} \hat{a}_{l i}+\rho_{A_{i}}\left(\sum \dot{q}_{1}\right) \hat{a}_{l i}
$$

where $\rho_{A}$ is the length between distal and center of mass with $A_{l}^{0}$ are points at the joints and $A_{i}^{*}$ are points at the center of mass of a segment. And linear accelerations of center of mass are, 


$$
\begin{aligned}
{ }^{N} \vec{a}^{A_{i}^{*}}= & \left(-l_{A_{i-1}}\left(\sum \dot{q}_{i-l}\right)^{2} \widehat{a}_{2(i-l)}+l_{A_{i-1}}\left(\sum \ddot{q}_{i-1}\right) \hat{a}_{I(i-l)}\right) \\
& +\left(-\rho_{A_{i}}\left(\sum \dot{q}_{i}\right)^{2} \hat{a}_{2 i}+\rho_{A_{i}}\left(\sum \ddot{q}_{i}\right) \hat{a}_{l i}\right) .
\end{aligned}
$$

Using Kane's method, the angular velocities and the velocities of points can be factored out to obtain partial angular velocities and partial velocity vectors. To factor these velocities, quantities called $u_{i} \equiv \dot{q}_{i},(i=1,2,3, \ldots, 6)$ are introduced. $u_{1}$ is known as the $i$-th generalized speed of the system. For example, the angular velocity of segment $A_{1}$ with respect to reference frame is derived as,

$$
{ }^{N} \vec{\omega}^{A_{1}}=\left(\hat{a}_{31}\right) u_{1}+(\overrightarrow{0}) u_{2}+(\overrightarrow{0}) u_{3}+(\vec{O}) u_{4}+(\overrightarrow{0}) u_{5}+(\overrightarrow{0}) u_{6}
$$

The partial angular velocity of the first segment with respect to reference frame $\mathrm{N}$ is obtained from the above expression,

$$
{ }^{N} \vec{\omega}_{1}^{A_{l}}=\widehat{a}_{31} .
$$

Likewise, the partial linear velocity can also be obtained from the linear velocity of segments. Using Kane's method there is no need to compute partial velocities at the joints because the joint interaction forces are noncontributory.

Generalized active forces and generalized inertia forces are then formulated for the segments. To form the generalized active forces, vector dot products between the partial velocities of points and the forces acting at those points are computed and added together. Additionally, dot products between partial angular velocities and torques are added and summed together with the previous result. The generalized active forces, $F_{i}$, are obtained as,

$$
\begin{aligned}
F_{i}= & \left({ }^{N} \vec{v}_{i}^{A_{1}{ }^{*} \cdot} \cdot-m_{A_{l}} g \hat{n}_{2}\right)+\left({ }^{N} \vec{v}_{i}^{A_{2}{ }^{*} \cdot} \cdot-m_{A_{2}} g \hat{n}_{2}\right)+\left({ }^{N} \vec{v}_{i}^{A_{3}{ }^{*} \cdot-}-m_{A_{3}} g \hat{n}_{2}\right)+ \\
& \left({ }^{N} \vec{v}_{i}^{A_{4}{ }^{*} \cdot} \cdot-m_{A_{4}} g \hat{n}_{2}\right)+\left({ }^{N} \vec{v}_{i}^{A_{5}{ }^{*} \cdot} \cdot-m_{A_{5}} g \hat{n}_{2}\right)+\left({ }^{N} \vec{v}_{i}^{A_{6}{ }^{*} \cdot} \cdot-m_{A_{6}} g \hat{n}_{2}\right)+ \\
& \left({ }^{N} \vec{v}_{i}^{A^{o}} \cdot F\right)+{ }^{N} \vec{\omega}_{i}^{A_{1}}\left(\tau_{N / A_{l}}-\tau_{A_{l} / A_{2}}\right)+{ }^{N} \vec{\omega}_{i}^{A_{2}}\left(\tau_{A_{l} / A_{2}}-\tau_{A_{2} / A_{3}}\right)+ \\
& { }^{N} \vec{\omega}_{i}^{A_{3}}\left(\tau_{A_{2} / A_{3}}-\tau_{A_{3} / A_{4}}\right)+{ }^{N} \vec{\omega}_{i}^{A_{4}}\left(\tau_{A_{3} / A_{4}}-\tau_{A_{4} / A_{5}}\right)+ \\
& { }^{N} \vec{\omega}_{i}^{A_{5}}\left(\tau_{A_{4} / A_{5}}-\tau_{A_{5} / A_{6}}\right)+{ }^{N} \vec{\omega}_{i}^{A_{6}}\left(\tau_{A_{5} / A_{6}}\right)
\end{aligned}
$$

for $i=1,2,3, \ldots, 6$ where $\mathrm{m}$ is the mass of each segment, $g$ is the gravitational force, $\hat{n}$ is the mutually orthogonal unit vectors respected to a reference frame $N$ and $\tau$ is the inertial torque of a body in $N$. After computing the generalized active forces, the generalized inertia forces are calculated next. These are composed of the dot products between the partial velocities of the mass centre and the inertial forces there, as well as the dot products between the partial angular velocities and the inertial torques. The generalized inertial forces represented by the equations are formulated as, 


$$
\begin{aligned}
& F_{i}^{*}=\left({ }^{N} \vec{v}_{i}^{A_{l}{ }^{*}} \cdot-m_{A_{l}}{ }^{N} \vec{a}^{A_{l} *}\right)+\left({ }^{N} \vec{v}_{i}^{A_{2}{ }^{*}} \cdot-m_{A_{2}}{ }^{N} \vec{a}^{A_{2} *}\right)+\left({ }^{N} \vec{v}_{i}^{A_{3}{ }^{*}} \cdot-m_{A_{3}}{ }^{N} \vec{a}^{A_{3} *}\right) \\
& +\left({ }^{N} \vec{v}_{i}^{A_{4}{ }^{*} \cdot} \cdot-m_{A_{4}}{ }^{N} \vec{a}^{A_{4} *}\right)+\left({ }^{N} \vec{v}_{i}^{A_{5} *} \cdot-m_{A_{5}}{ }^{N} \vec{a}^{A_{5} *}\right)+\left({ }^{N} \vec{v}_{i}^{A_{6}{ }^{*}} \cdot-m_{A_{6}}{ }^{N} \vec{a}^{A_{6} *}\right) \\
& +{ }^{N} \vec{\omega}_{i}^{A_{I}}\left(-I_{A_{l} *}{ }^{N} \vec{\alpha}^{A_{l}}\right)+{ }^{N} \vec{\omega}_{i}^{A_{2}}\left(-I_{A_{2} *}{ }^{N} \vec{\alpha}^{A_{2}}\right)+{ }^{N} \vec{\omega}_{i}^{A_{3}}\left(-I_{A_{3} *}{ }^{N} \vec{\alpha}^{A_{3}}\right) \\
& +{ }^{N} \vec{\omega}_{i}^{A_{4}}\left(-I_{A_{4} *}{ }^{N} \vec{\alpha}^{A_{4}}\right)+{ }^{N} \vec{\omega}_{i}^{A_{5}}\left(-I_{A_{5} *}{ }^{N} \vec{\alpha}^{A_{5}}\right)+{ }^{N} \vec{\omega}_{i}^{A_{6}}\left(-I_{A_{6} *}{ }^{N} \vec{\alpha}^{A_{6}}\right)
\end{aligned}
$$

for $i=1,2,3, \ldots, 6$ where $I$ is the moment of inertia of body $A_{i}$ about an axis parallel to its angular aeleration and pasing through its center of body mass.

\subsection{Data collection}

The kinematic data for a subject's segment angles are obtained from the sample data of walking, running and vertical jumping activities captured using Vicon Nexus 1.3 system. The system consists of 12 Vicon MX-3+ cameras, 12 Vicon MX-F40 cameras, two DV $(50 \mathrm{~Hz})$ cameras and a force platform $(100 \mathrm{~Hz})$ as shown in Fig. 2.

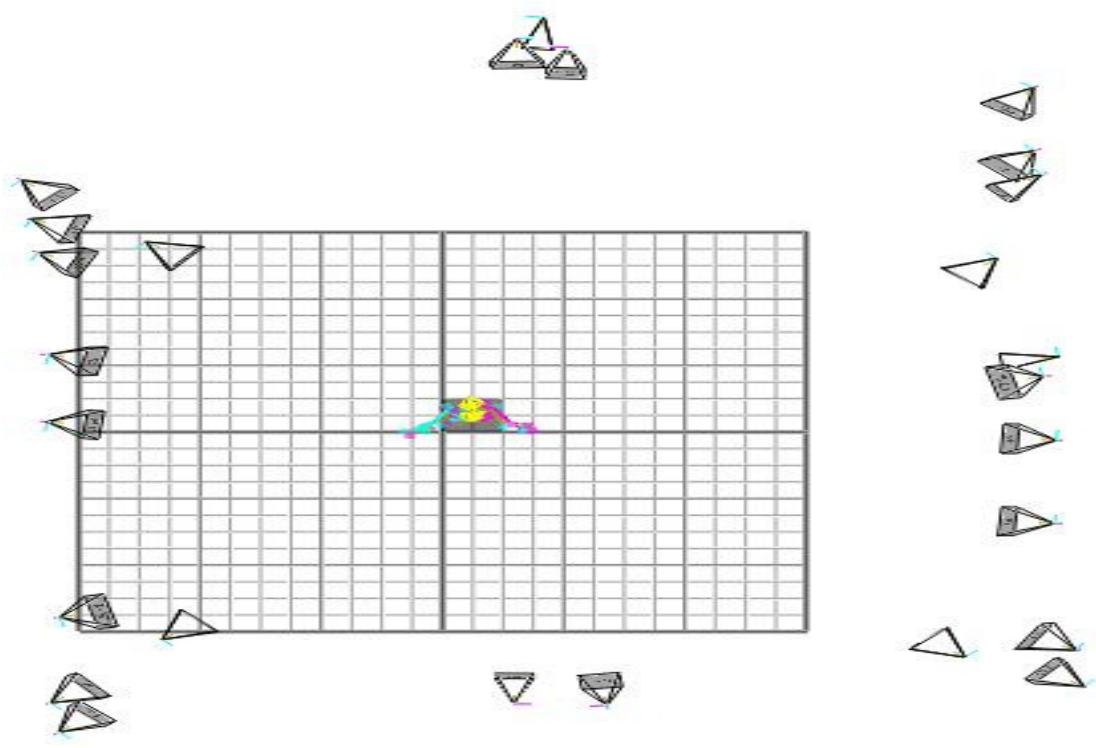

Fig. 2 Recording space for capturing data of human movement

To validate the model, data from walking, running and jumping activities were selected for analysis. In walking and running activities, it is assumed that force occurs when the foot touches the ground, thus in the swinging phase forces are not calculated. The kinematics and anthropometric data of subject are taken as input and applied to the dynamics equation obtained in the previous section to calculate the torques of each segment. 


\section{Measurement of Physical Subject}

The weight and height of subject were measured to allow torque calculation. The anthropometric data are used to calculate distal and proximal lengths from its center of mass as well as the mass of each segment.

\subsection{Measurement of segment length and the length between proximal and center of mass for each segment}

The subject is $176 \mathrm{~cm}$ of height and $75 \mathrm{~kg}$ of weight. The length of each segment is

$$
\begin{aligned}
& l_{A_{l}}=0.300 \text { (length of foot segment, } A_{1} \text { ) } \\
& l_{A_{2}}=0.553 \text { (length of calf segment, } A_{2} \text { ) } \\
& l_{A_{3}}=0.605 \text { (length of thigh segment, } A_{3} \text { ) } \\
& l_{A_{4}}=0.605 \text { (length of trunk segment, } A_{4} \text { ) } \\
& l_{A_{5}}=0.361 \text { (length of upper arm segment, } A_{5} \text { ) } \\
& l_{A_{6}}=0.483 \text { (length of forearm segment, } A_{6} \text { ). }
\end{aligned}
$$

The length between distal and center of mass of each segment is denoted by notation $\rho_{A_{i}}(i=1,2, \ldots, 6)$. The calculation of segments length are based on standard anthropometric data from Ref. 6 ,

$$
\begin{aligned}
\rho_{A_{l}} & =\left(\text { length of foot segment } A_{l}\right) \times\left(\text { position of center of mass } A_{l}^{*}\right) \\
& =(0.300 \times 0.500) \mathrm{m} \\
& =0.150 \mathrm{~m} \\
& =0.132 \mathrm{~m} .
\end{aligned}
$$

\subsection{Measurement of mass of each segment}

Mass for each segment is denoted by $m_{A_{i}}(i=1,2, \ldots, 6)$. Measurement of the mass of each segment is based on the mass of the subject and the mass proportion of each segment. For example, mass of the first segment,

$$
\begin{aligned}
m_{A_{l}} & =(\text { mass of the subject }) \times\left(\text { the mass proportion of segment } A_{1}\right) \\
& =(75 \times 0.0145) \mathrm{kg} \\
& =1.087 \mathrm{~kg} .
\end{aligned}
$$

The values of mass proportion for each segment are obtained from the anthropometric data.

\subsection{Measurement of moment of inertia at center of mass of each segment}

Moment of inertia at the center of mass of each segment is denoted by $I_{A_{i}^{*}}(i=1,2, \ldots, 6)$. Calculation of moment of inertia is,

$$
\begin{aligned}
I & =\frac{m}{2}\left(x-\rho_{o}\right)^{2}+\frac{m}{2}\left(x+\rho_{o}\right)^{2} \\
& =m \rho_{o}^{2}+m x^{2}
\end{aligned}
$$


where,
$m$ = mass of segment
$\rho_{o} \quad=$ radius of gyration
$x=$ distance between center of mass and distal of segment for each segment.

The radius of gyration values are obtained from the anthropometric data. The length of center of mass and distal segment were obtained in Section 3.1 and the values of the mass segment obtained from the calculation in Section 3.2. From the above equation, the moment of inertia at the center of mass of each segment are as follows,

$$
\begin{aligned}
I_{A_{1} *} & =\left(1.087 \times 0.143^{2}\right)+\left(1.087 \times 0.150^{2}\right) \mathrm{kgm}^{2} \\
& =0.047 \mathrm{kgm}^{2} .
\end{aligned}
$$

\section{Dynamics Equations}

Kinematic and anthropometric values obtained in section 3 are substituted into the dynamic equation,

$$
F_{i}+F_{i}^{*}=0
$$

for $i=1,2,3, \ldots, 6$. The dynamics equation can be represented in the matrix form

$$
M \overrightarrow{\ddot{Q}}=\vec{G}+\vec{E}+\vec{T}
$$

where

$M$ : mass matrix

$\overrightarrow{\ddot{Q}}$ : angular acceleration vectors

$\vec{G}$ : vector of moments from gravitational forces

$\vec{E}$ : vector of moments from external forces, and

$\vec{T}$ : vector of applied torques.

\section{Results and Discussion}

The torque of the joints is obtained from the model developed above by using the kinematic data obtained from walking, running and vertical jumping activities. The determination of torques at the joint segment in the human body will help physicians, researchers and orthopedic surgeon to identify mechanisms of movement of the body, to verify the effectiveness of optimization and to design an implant, respectively. Therefore, researchers can perform motion analysis to optimize, prevent or reduce the occurrence of injuries.

Figure 3, 4 and 5 show the values of torque at each joint of body segments at the end of foot (toe), ankle, knee, hip, shoulder and elbow joints during walking, running and jumping activities. From the figures, the greatest values of torques occur at the end of the foot segment (toe) and the ankle joint for the weight of the body are projected at the segments nearest to the ground. From Fig. 3, results obtained are similar to the force of 
$\left(F_{y}\right)$ walking patterns, specifically the 'M-shaped' patterns, while Fig. 4 shows quite a similar running pattern obtained by Ref. 7 .

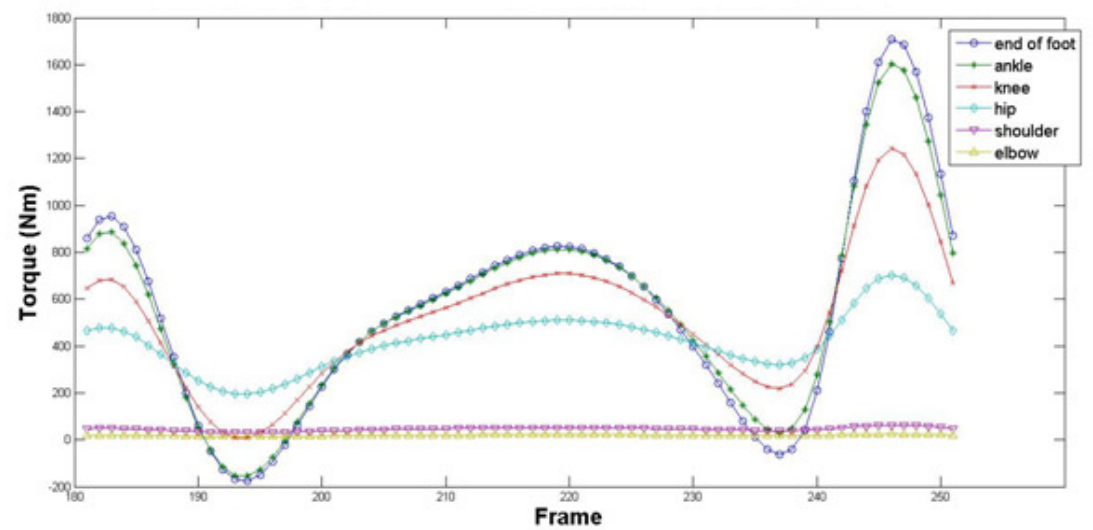

Fig. 3 The value of torque at each joint during walking activity in one gait cycle

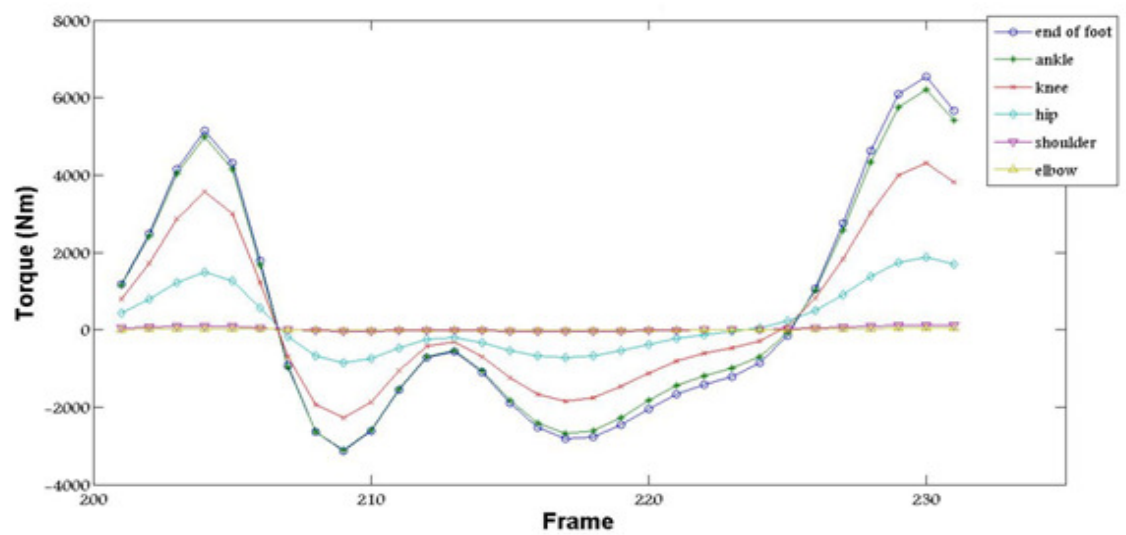

Fig. 4 The value of torque at each joint during running activity

Figure 5 shows the torque value for movement jump activity. Similar to movements of walking and running, the highest torque values occur at the segment nearest to the ground. During performance of jumping, changes of torques occur significantly during takeoff and landing phases. The information helps to alleviate the risk of injury during landing performance from jumping. The recorded activity enables the determination of torque through the application of dynamic modeling that has been developed to optimize the movement and prevent the occurrence of injuries. 


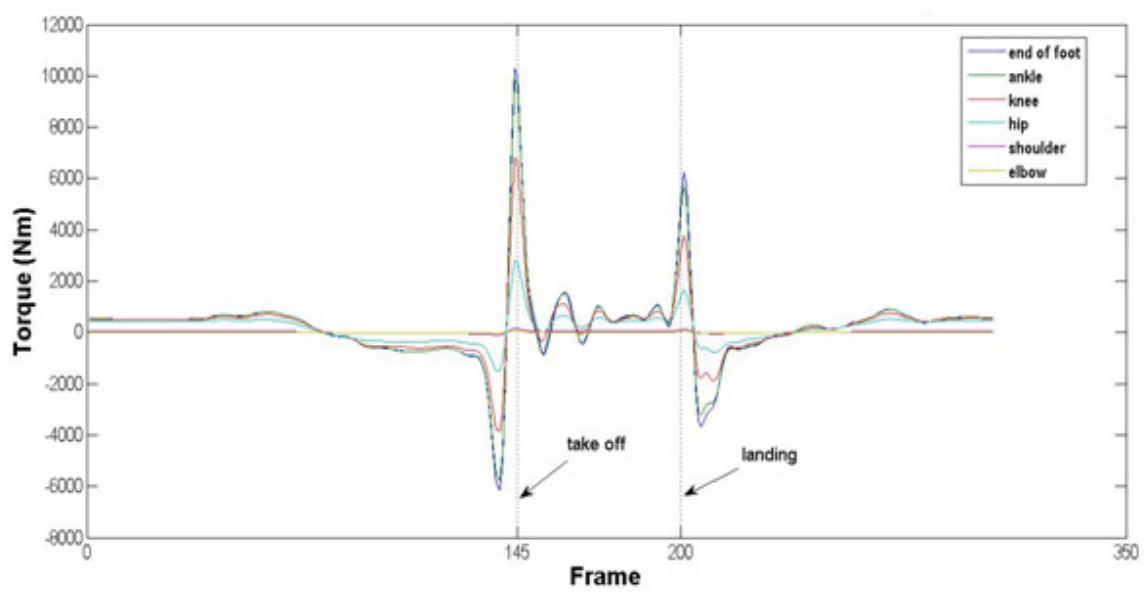

Fig. 5 The value of torque at each joint during a jumping activity

\section{Conclusion}

The paper discusses a development of a kinematics chain model that represents a human movement. This work is important because it shows that a Vicon Nexus system coupled with Kane's method provides people with information about where their greatest torque is when they perform an activity such as walking, running and jumping vertically. This research can be applied to other people in situations like rehabilitation of injury or stroke victims or fine tuning of walking or running styles for professional athletes. Thus determination of torque through the application of dynamic modeling is possible to optimize the movement and prevent the occurrence of injuries.

\section{Acknowledgments}

The authors are gratefully acknowledge the Financial supports received in the form of research university grants (UKM-GUP-BTT-07-25-174) from Universiti Kebangsaan Malaysia and (UKM-ST-07-FRGS0027-2009) from the Ministry of Higher Education, Malaysia.

\section{References}

1. B. R. Gillespie, Kane's equation for haptic display of multisegment systems, Haptics-e, 3, 2 (2003).

2. A. Nagano, T. Komura, S. Fukashiro and R. Himeno, Force, work and power output of lower limb muscles during human maximal-effort countermovement jumping, J. Electromyography and Kinesiology 15, 367 (2004).

3. D. Glaser, A. D. Douglas, D. K. Richard and M. M. Todd, In vivo comparison of hip mechanics for minimally invasive versus traditional total hip arthroplasty, J. Clinical Biomechanics 23(2), p. 127 (2007). 
4. F.H.M. Ariff and A. S. Rambely, Determination of torques at upper limb joints during jumping in badminton smash via Kane's Method, in Proc. of 26th Int. Symp. on Biomechanics in Sports, pp. $73-76$ (2008).

5. S. A. Hummel and M. A. Hubbard, Musculoskeletal Model for Backhand Frisbee Throws, in 8th Int. Symp. on Comput. Simulation in Biomechanics (The Politecnico di Milano,Milan Italy, 2001).

6. D. A. Winter, Biomechanics of Human Movement, (John Wiley and Sons, New York, 1979).

7. A. E. Minetti, The biomechanics of skipping gaits: a third locomotion paradigm?, The Royal Soc. 265, 1227 (1998). 